\title{
On the Impact of the Customer Base on the Added Value through System-Oriented Service Delivery in Industrial Maintenance
}

\author{
Clemens Wolff \\ Karlsruhe Institute of Technology \\ clemens.wolff@kit.edu
}

\author{
Melanie Reuter-Oppermann \\ Karlsruhe Institute of Technology \\ melanie.reuter@kit.edu
}

\author{
Niklas Kühl \\ Karlsruhe Institute of Technology \\ niklas.kuehl@kit.edu
}

\begin{abstract}
Today, during service delivery, providers allocate their delivery resources such that their own delivery-dependent costs are minimal. However, during service delivery, costs arise not only for the provider, but for the customer, too. In industrial maintenance, for example, those costs arise-depending on service delivery - due to longer equipment unavailability.

The concept of system-oriented service delivery aims at minimizing the total (customer and provider) delivery-dependent costs within the service system and promises a Pareto improvement over today's practice. Hence, added value is created. However, so far, we have no understanding of the magnitude of and factors favoring high added value through system-oriented service delivery.

Consequently, this work aims at filling this gap and widening knowledge on the added value through system-oriented service delivery. We present a simulation study to elaborate on the added value in dependency of the customer base constellation in an industrial maintenance illustrative scenario.
\end{abstract}

\section{Introduction}

Within the field of service science, Ostrom et al. [1, 2] identify the need for further research in service delivery. Recently, researchers evaluated service delivery approaches within service systems [3]. In detail, the authors argue that all system participants-i.e. provider and customers-have delivery-dependent consequential costs associated to a specific service delivery solution. Today, providers allocate delivery resources such that their own delivery-dependent costs are minimized (including possible service level agreement penalties). This implies that providers favor any delivery solution that results in minor cost savings for themselves, even if much larger additional costs are introduced on the customer side. Hence, the authors stress a systematic resource allocation inefficiency from a system viewpoint. Wolff et al. [3] refer to this as provider-oriented service delivery.

Consequently-following the idea of Service Systems [4] and Service Systems Engineering [5]-Wolff et al. [3] propose the concept of system-oriented service delivery that can be summarized by two components: First, a shift in the delivery objective. Instead of delivering such that the provider's delivery costs are minimal, providers deliver service such that the system's total delivery-dependent costs are minimized. In other words, the provider takes delivery-dependent costs on both, the provider, as well as on the customer side in account during resource allocation. Second, the introduction of a monetary re-allocation method allowing monetary compensation and cash flow between participants. Given the monetary re-allocation mechanism, individual advantages and disadvantages through the shift from current delivery approaches towards system-oriented service delivery are compensated and the added value is distributed among participants. Note that-per definition-any disadvantages can be fully compensated, hence, system-oriented service delivery results in a Pareto improvement (i.e. at least one participant has an advantage and no participant has a disadvantage through the new solution) over provider-oriented service delivery. The improvement compared to today's solution (from a system viewpoint) is referred to as added value.

Whilst the added value is greater or equal to zero, the magnitude of added value through the shift towards system-oriented service delivery cannot be quantified on a general basis. However, there may still be a pattern that allows for indications on the added value on a general basis. For example, as the added value is created by a shift in resource allocation, and, consequently, a shift in service delivery response time between customers, the added value depends greatly on the differences in response time sensitivity of delivery-dependent costs on the customer 
side. It is evident that the composition of the customer base with regard to their delivery-dependent costs plays a significant role in the creation of added value through system-oriented service delivery. This can easily be illustrated by a simple example: If two customers' delivery-dependent costs are equally sensitive to changes in the response time (i.e. have the same development of their delivery-dependent costs over an increased response time), no value can be gained by interchanging their priority, as savings of one customer introduce additional expenses for the other customer to the same extend. Consequently, the cost saving potential is low. However, if their delivery-dependent costs are differently response time sensitive, the gain for one customer may be higher than the loss for the provider and other customer and added value is created, resulting in a high cost saving potential. Taking this simple example as a basis, we believe that the amount of added value created depends highly on the range of the customers' delivery-dependent cost time-sensitiveness.

Consequently, the goal of this work is twofold: First, we aim at addressing the lack of knowledge on the magnitude of added value created through system-oriented service delivery. Furthermore, second, we aim at evaluating initial thoughts on the range of delivery-dependent cost time-sensitiveness expressed in the above example. As the quantification of the added value is impossible on an abstract level, we use industrial maintenance as an illustrative scenario [6] and apply a simulation experiment within this domain.

The remainder of this work is structured as follows: In Section 2, we introduce fundamentals and related work. In Section 3, we discuss how the customer base can be modeled in order to evaluate its impact on the the added value through system-oriented service delivery. In Section 4, we present a provider- and system-oriented technician dispatching problem as basis for the simulation study. Furthermore, we present the simulation model, experiment, and its results. Finally, in Section 5, we conclude this study and point out limitations and future work.

\section{Fundamentals and Related Work}

In this section, we introduce fundamentals and related work. We elaborate on industrial maintenance, technician dispatching, as well as on system-oriented service delivery.

\subsection{Fundamentals of Industrial Maintenance}

Industrial maintenance is a prominent example of a service within the industrial domain [7]. According to Geraerds [8], maintenance includes "all activities aiming at keeping an item in, or restoring it to, the physical state considered necessary for the fulfillment of its production function". Research shows that manufacturers see efficient maintenance as an advantage over their competitors [9].

Currently, the field of industrial maintenance undergoes two major changes: First, maintenance strategies are shifting from reactive towards preventive maintenance strategies. In other words, instead of running equipment until it fails, one tries to prevent its failure in the first place [10]. Second, maintenance business models change. Instead of selling individual maintenance tasks, providers aim at selling long-term maintenance engagements [11], often realized through full service (e.g. $[12,13]$ ), availability-based (e.g. [14]), and performance-based (e.g. [15]) maintenance contracts.

Regardless of maintenance strategy or service offering, one core task in operations (i.e. service delivery) remains the same: Providers need to schedule service tasks given their limited workforce, a task referred to as task scheduling or technician dispatching. During assignment, providers usually aim for two contradictory goals: high profitability and technician utilization, and high customers satisfaction, which is usually measured in short-term responsiveness [16]. In the following section, we further elaborate on the technician dispatching problem from an operations research perspective.

\subsection{Fundamentals of Technician Dispatching}

The technician dispatching problem marks an extension to the vehicle routing problem initially introduced by Dantzig and Ramser [17], as it adds the further complexity of task duration at the customer's site. In literature, technician dispatching is addressed under multiple terms, ranging from technician or task scheduling to technician dispatching. Literature on technician dispatching can be separated into two, non-disjunctive streams: The first stream focuses on the formulation of technician dispatching optimization problem and includes work on specific use cases (e.g. $[18,19,20])$ as well as literature reviews (e.g. [21, 22]). The second stream centers around heuristics solving formulated dispatching problems (e.g. [23, 24, 25]).

\subsection{Industrial Cost of Downtime}

The field of downtime cost elaborates on the costs associated with the downtime of a production-critical infrastructure. Evidently, this scenario can be looked at from multiple perspectives, hence, it is no surprise 
that costs of downtime are researched from multiple domains, as, for example, costs encountered during an electric outage (e.g. [26]), IT infrastructure downtime (e.g. [27]), or downtime of industrial production equipment. In the industrial sector, around $80 \%$ of manufacturers cannot predict their costs of downtime accurately, as estimated costs are exceeded by a factor between two and three [28].

Within cost of downtime, researchers commonly differentiate between direct and indirect costs of downtime [29]. The first refers to all costs that are directly related to the repair of the asset, common examples are the labor and material spent to repair the asset. The ladder refers to consecutive costs of a failure and include productivity loss (e.g. idle labor force), opportunity costs due to lost production outcome, or band aid costs (costs to minimize damage).

Fox et al. [30] determine the financial impact of machine downtime on the letter sorting process in the Australia Post. They use historic data and come to the conclusion that the loss of production capacity has the highest impact. Another study by Edwards et al. [31] presents a regression model to estimate costs associated to machine failure in the mining industry. Their model is trained on historic data. Furthermore, Vegunta and Milanovic [32] present a framework to estimate costs of downtime within industrial production processes due to short-time voltage sags. Wolff and Schmitz [33] contribute to this topic by pointing out further research need within the cost of downtime estimation and by presenting a high level approach to determine opportunity costs associated with machine downtime.

\subsection{System-Oriented Service Delivery}

System-oriented service delivery was first introduced by Wolff et al. [3]. The authors highlight a systematic resource allocation inefficiency during today's resource allocation practice within service systems-what they refer to as provider-oriented service delivery-as providers aim at minimizing their own delivery-dependent costs without taking delivery-dependent costs on the customer side into account. Following the ideas behind Service Systems Engineering [5] and Service Systems (e.g. [34, 4]), the authors propose the formation of one holistic service system including all customers and within that service system resource allocation such that the total delivery-dependent system costs-hence, the sum of delivery-dependent costs on the customer as well as on the provider side-are minimized. Per definition, overall delivery-dependent costs must be lower (or equal), hence-from a system perspective-added value is created. However, from an individual participant's perspective, the shift in resource allocation approach may result in participants having an advantage (i.e. a shorter response time than before), and others having a disadvantage (i.e. a longer response time than before). In order to prevent disadvantages on an individual participant level, the authors propose a monetary re-allocation mechanism through which any advantages and disadvantages of all participants are shared among others. Given the non-negative added value, any participant's individual disadvantage can be compensated, and the remaining added value can be distributed among all participants. Given the monetary re-allocation mechanism, the authors are able to show that the proposed approach results in a Pareto improvement over today's solution. The findings from system-oriented service delivery furthermore support the idea of value co-creation, as value is created through the interaction of multiple players [35, 36]. All technician dispatching problems introduced in Section 2.2 follow the provider-oriented delivery approach.

Given first conceptional work on system-oriented service delivery, there are still many open questions, some concerning system design, others its implementation in practice: For system-oriented service delivery, for example, customers must know their delivery-dependent costs, which is a challenging tasks in a variety of domains (e.g. [27] for IT infrastructure). Furthermore-from a mechanism design perspective-we must ensure incentive compatibility. In other words, all participants should have the incentive to provide their true delivery dependent costs. This is important as exaggerated delivery-dependent costs may result in faster service delivery, i.e. a shorter response time. This, however, does not align with the core idea of minimizing true total delivery-dependent system costs. We believe that incentive compatibility can be achieved by carefully designing the distribution of added value, which, itself, marks another unanswered question in the field of system-oriented service delivery. In addition, we must further understand the magnitude of added value created through the shift towards system-oriented service delivery. So far, it has only been shown that system-oriented service delivery results in a Pareto improvement over provider-oriented service delivery, however, we have no understanding on the magnitude of added value created. Furthermore, we would like to be able to identify markets in which the application of system-oriented service delivery is especially promising. Last but not least, providers must redesign their value-proposition within system-oriented service delivery, and, consequently, perform business model 
innovation according to Maglio and Spohrer [37].

As the ratio of delivery resources and service demand is not changed, it is evident that the added value is achieved by a resource re-allocation among customers. Additional value is created through the shift of delivery resources at a specific time from one customer to another, if-and only if-the savings introduced for the customer through the resource re-allocation are greater than the additional expenses of the other participants. Hence, it is evident that the magnitude of added value depends greatly on the differences in delivery-dependent costs of the participants between the provider- and the system-oriented resource allocation.

Consequently, we aim at further exploring the impact of the service system composition with regard to the delivery-dependent costs on the added value created through system-oriented service delivery.

\section{Modeling Cost of Downtime over the Customer Base}

The service system is composed of many customers and-for simplification-only one provider. However, the following argumentation also holds true for service systems with more than one provider. The customers within the service system-the customer base-jointly influence the added value created through the shift towards system-oriented service delivery through their composition with regards to their delivery-dependent costs. Given the goal of this work, we first introduce how cost of downtime is modeled for an individual customer. Second, we move from modeling one customer to modeling an entire customer base, hence, the composition of the service system with regards to the customers. Note that in this context, one customer does not refer to a business itself (i.e. the manufacturer), but instead to an equipment unit (i.e. a production equipment unit) requiring service.

\subsection{Modeling the Delivery-Dependent Costs of an Individual Customer}

Given previous notion, researchers differentiate between direct and indirect costs of downtime. In the context of system-oriented service delivery, indirect costs of downtime refer to the delivery-dependent costs faced by the customers. It is evident that indirect costs of downtime in industrial manufacturing increase over time, as, for example, a higher equipment unavailability results in higher production time losses. For simplification, we assume a linear relationship between the delivery-dependent costs and the response time. In case of an immediate repair, delivery-dependent costs are 0 . Hence, delivery-dependent costs for one customer can be modeled by the cost slope $s$ which represents the sensibility of their costs of downtime (i.e. delivery-dependent costs) on the response time.

\subsection{Modeling the Delivery-Dependent Costs of the Customer Base}

If an individual customer's cost of downtime can be modeled using a slope $s$, the entire customer base can be modeled using a distribution representing $s$ over all customers.

In order to understand the impact of the customer base on the added value created, we use three different distributions to model typical customer bases within industrial maintenance: a normal, uniform, and mixture distribution of two symmetric normal distributions.

The normal distribution models a homogeneous customer base with regards to their cost slope, as customers slope centers around a mean value. In this case, the maintenance provider only serves one customer segment with regards to their cost slope. The uniform distribution models the contrary, hence, heterogeneous customer base. This case corresponds to a maintenance provider serving many different customers with regard to their cost slope. In other words, the response time sensitivity of the customer's cost of downtime differs between all customers. Finally, the mixture distribution corresponds to customer bases that consist of two customer segments with regards to their cost of downtime response time sensitivity. A common example of such a customer base is the automotive market, where some customers (usually the car manufacturers) have highly automated and integrated production systems with a very high response time sensitivity, whereas other customers (usually their suppliers) rely on "demand-based" production that is less response time sensitive as their production system is less integrated.

For the three slope distributions, an exemplary visualization is provided in Figure 1. One row corresponds to one customer base, in which the left diagram shows the distribution of cost slopes $s$ and the right figure indicated the cost of downtime development over response time for exemplary and randomly drawn customers from that customer base. In the top distribution - the normal distribution-the customers cost of downtime develop rather similarly to each other. In the second case, the uniform distribution, we see clear differences of the cost of downtime development over the response time. In the third case-the mixture distribution-we see two segments of customers having roughly equal cost of downtime development within 

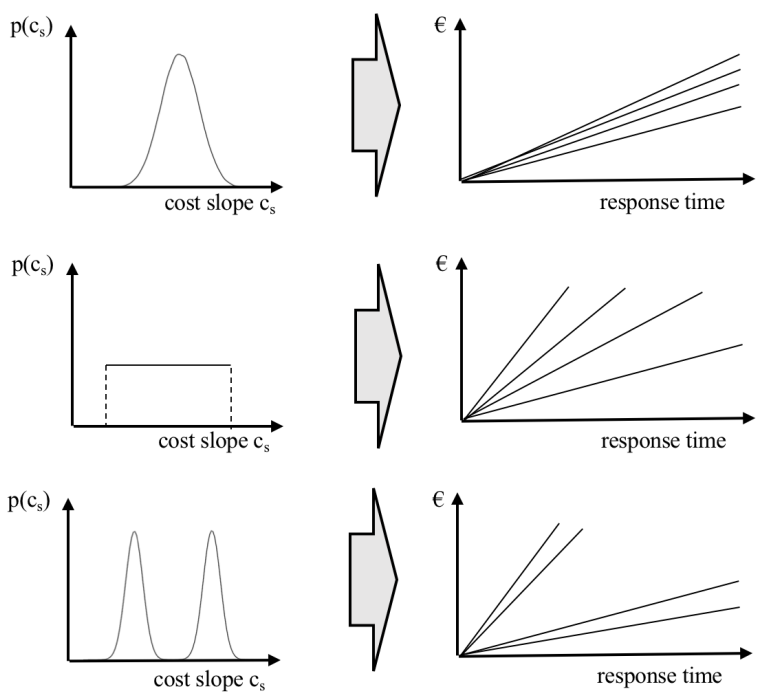

Figure 1. Exemplary slope distribution and their cost of downtime over response time.

one segment, and highly different cost of downtime development over response time between the segments.

\section{Elaborating on the Added Value for Different Customer Base Patterns}

Based on the introduced distributions to model different customer bases, we conduct a simulation experiment to further elaborate on both, the added value created and the impact the customer base has on the added value created through system-oriented service delivery. A simulation experiment is a promising approach to test many samples of the assumed cost slope distributions [38].

In this section, we first introduce a provider- and system-oriented technician dispatching problem (as resource allocation mechanism). Second, we present the core components of the simulation model. Third, we list parameters and scenarios used for the simulation study. Finally, we present results and discuss those.

\subsection{Technician Dispatching Problem}

In this work, the technician dispatching problem is limited to the assignment of a set of tasks to a set of technicians over the period of one day. Tasks are assigned under the consideration of hard work time restrictions (no overtime allowed) and routing. Furthermore, not all tasks must be assigned, hence, a backlog of tasks is possible. In practice, those tasks would be added to the set of tasks for following days. For simplification, technician and required task skills are neglected.
Given the set $T=\{1, \ldots, m\}$ of technicians (and for easier understanding, also their home locations) and set $W=\{1, \ldots, n\}$ of tasks (again, also their locations), the technician dispatching problem can be modeled by a Graph $G=(V, E)$. The set of vertexes $V$ consists of the technician and task locations, hence, $V=\{T, W\}$. The set $E$ represents edges between any two vertexes, hence, $E=\{(i, j)\}$ with $i, j \in V$. Each edge from $i$ to $j$ with $i, j \in V$ has a travel time $t_{i j}$ associated with it. A task $i \in W$ has a duration of $d_{i}$. The maximum work time for a technician is provided by $t_{m}$ and does not vary among technicians. Furthermore, all technicians start their shift at the same time, in detail, at $t_{s} . f$ and $p$ refers to technician travel costs per time unit and a penalty for the provider, if a task is not performed that day, respectively.

For the optimization problem, we define the following two decision variables: First, $a_{i}$ denotes the schedules start time ( 0 indicating immediate start). Second, we define $x_{i j t}, i, j \in V, t \in T$ as shown in 1 .

$$
x_{i j t}= \begin{cases}1 & \text { if } \mathrm{t} \text { travels directly from } \mathrm{i} \text { to } \mathrm{j} \\ 0 & \text { otherwise }\end{cases}
$$

Given the defined variables, the provider-oriented dispatching problem is formulated as shown in 2 . The objective function for provider-oriented technician dispatching problem-as shown in $2 \mathrm{a}$-minimizes provider costs that are limited to routing and penalty costs. According to Sörensen et al. [39], routing costs are a significant cost driver in field services.

$$
\begin{aligned}
& \min _{a, x} \sum_{i \in V} \sum_{j \in V} \sum_{t \in T} t_{i j} x_{i j t} f+\left(n-\frac{1}{2} \sum_{i \in V} \sum_{j \in V} \sum_{t \in T} x_{i j t}\right) p \\
& \text { s.t. } \sum_{i \in V \backslash\{j\}}\left(x_{i j t}-x_{j i t}\right)=0 \quad \forall j \in V, t \in T \text {, } \\
& \sum_{j \in W} x_{i j t} \leq 1 \quad \forall i, t \in T, \\
& \sum_{j \in W} x_{i j t}=0 \quad \forall i \in T, t \in T \backslash\{i\}, \\
& \sum_{i \in V} \sum_{t \in T} x_{i j t} \leq 1 \quad \forall j \in W \\
& \left(t_{s}+t_{i j}\right) x_{i j t} \leq a_{j} \quad \forall i, j \in W, t \in T, \quad \text { (2f } \\
& \left(a_{i}+d_{i}+t_{i j}\right) x_{i j t} \leq a_{j} \quad \forall i, j \in W, t \in T, \quad \text { (2g) } \\
& \left(a_{i}+d_{i}+t_{i j}\right) x_{i j t} \leq b_{s}+t_{m} \forall i, j \in W, t \in T, \quad \text { (2h) } \\
& x_{i j t} \in\{0,1\} \quad \forall i, j \in V, t \in T
\end{aligned}
$$

The formulation shown in 2 follows common formulation approaches of the technician dispatching problem (e.g. [18, 20, 19]): Constraint 2b conserves flow, $2 \mathrm{c}$ and $2 \mathrm{~d}$ limit technician routes to one and ensure that each technician leaving also returns to his home 
depot. Given 2e, each task is only visited by one technician. Using $2 \mathrm{f}$ and $2 \mathrm{~g}$, technician arrival times are calculated and $2 \mathrm{~h}$ ensures a maximum working time. $2 \mathrm{i}$ imposes the binary decision variable.

In order to formulate the system-oriented technician dispatching problem, the objective function needs to be modified to correspond to the system's total delivery-dependent costs. Those include incurred costs of downtime on the customer side and travel costs on the provider side. Penalties are not further taken into account as they are total system cost neutral. The objective function for system-oriented technician dispatching is shown in 3 , whereas the constraint body is equivalent to the provider-oriented technician dispatching problem as shown in 2 .

$$
\min _{x, a} \sum_{i \in V} \sum_{j \in V} \sum_{t \in T} t_{i j} x_{i j t} f+\sum_{i \in W} a_{i} s_{i}
$$

\subsection{Simulation Model}

The simulation model is able to solve a dispatching problem twice, once following provider-, and once following system-oriented service delivery. After solving the optimization problems, the total system costs of the given solutions are calculated and compared. Hence, in other words, the simulation model solves one problem instance, and based thereon, calculates the added value through system-oriented service delivery. In detail, the simulation model consists of three modules, namely the problem set generator, the solver, and the cost calculator.

The first module, the problem instance generator, creates a set of tasks that need to be assigned to a set of technicians. During generation, the generator follows pre-defined distributions.

The second module, the solver, takes the problem set as input and solves it according to the provided delivery approach. Hence, this module is responsible for creating the actual technician schedules. The solver relies on heuristics and assigns tasks to technicians according to the technician dispatching problems introduced in Section 4.1. As the technician dispatching problem is NP hard, the solver relies on heuristic solving [25]. In detail, the solver relies on a two-step heuristic solving approach, by first creating a feasible solution using the Least Insertion Cost approach, and second, incrementally improving the feasible solution using a Variable Neighborhood Search. Both approaches are implemented according to Petrakis et al. [19].

The third module calculates the total delivery-dependent system costs for the given solutions, hence, for the provider- and system-oriented technician schedules. Based on the calculated system costs, the added value through the shift towards system-oriented service delivery is calculated.

Following Sargent's [40] suggestions on model validation, the simulation model was validated using conceptual validation. In detail-after development-the simulation experiment was presented to two researchers, one with a background in industrial maintenance and one with a background in simulation experiments. Furthermore, the study was presented to four experts from practice from two medium sized German production equipment manufacturers that also offer maintenance services to their customers. For each manufacturer, one dispatcher and one managing employee was consulted. In summary, feedback was concerned with the level of complexity of the optimization problem. In detail, two points were raised: First, the complexity of the model regarding constraints, as, for example, skills and task differentiation is not reflected in the optimization problem. Second, experts highlighted their concerns with the greenfield and offline scheduling approach. However, they also agreed that online scheduling may be neglected for now and be introduced in future work.

\subsection{Simulation Parameters, Experiment Factors, and Design of Experiment}

The objective of this work is the evaluation of the impact of the customer base on the added value created through system-oriented service delivery. In order to do so, a hierarchical two-factor design was chosen: The first factor $F 1$ refers to the overall variance found within the customer base, and the second factor F2 refers to the cost slope distribution used to model the customer base.

In order to keep the different distributions comparable, all distribution have the same mean cost slope of $\bar{s}=890$ following Salonen's [29] study in the Swedish manufacturing industry. Given the distributions introduced in section 3.2, the normal and uniform distribution are fully specified by a mean and variance (distribution parameters can be calculated accordingly). The mixture distribution, however, still has one degree of freedom left, as the variance of the underlying child distributions has not been specified. This is further shown in Figure 2 that displays three mixture distributions with the same overall variance and mean value, however, different standard deviations for the two underlying child normal distributions. We decided to use multiple instances of variance in order to allow for maximum insight.

In detail, we used the following scenarios to reflect the variation of the cost slope distribution: 


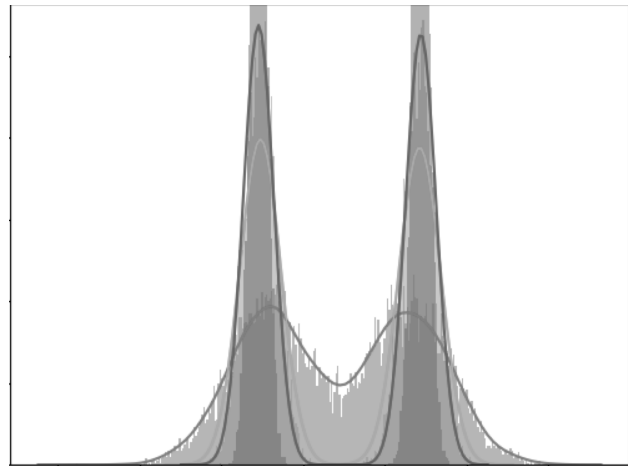

Figure 2. Different mixture distributions with equal mean and variance.

$$
\begin{aligned}
F 1=\{\operatorname{Var}(s) & =40,000 ; \operatorname{Var}(s)=90,000 ; \\
\operatorname{Var}(s) & =160,000 ; \operatorname{Var}(s)=250,000\}
\end{aligned}
$$

Furthermore-for factor two-the following distributions were used as scenarios:

$$
F 2=\{N, U, M 2, M 5, M 10\}
$$

In this case, $\mathrm{N}$ refers to the normal distribution, $\mathrm{U}$ to the uniform distribution, and $\mathrm{MX}$ to a mixture distribution of two symmetric normal distributions having a standard deviation of one $X$-th of the standard deviation as defined through factor one. In this case, $X$ indicates how well the two customer segments are separable (high $X$ indicating highly separable). Given those scenarios, there are $4 \cdot 5=20$ simulation cases that are each executed in a Monte Carlo Simulation. In a Monte Carlo Simulation, simulation models are executed repeatedly (here: $k=250$ times) in order to realize more reliable results under stochastic variance. Using a Monte Carlo Simulation for each case, a total of $20 \cdot 250=5000$ individual simulations are executed.

Table 1. Simulation experiment parameters.

\begin{tabular}{c|c}
\hline Parameter & Value \\
\hline Travel time (in min) & $\mathrm{N}(30,10)$ \\
Task duration (in min) & $\mathrm{N}(90,15)$ \\
Penalty $p$ (in monetary units) & 5000 \\
Number of tasks $n$ & 40 \\
Number of technicians $m$ & 10 \\
Shift start & $8 \mathrm{a} . \mathrm{m}$. \\
Work time $t_{\text {max }}$ (in min) & 480 \\
Travel time costs $f$ (in monetary units) & 5.53 \\
Neighborhood search iterations & 500 \\
\hline
\end{tabular}

For simulation, parameters as shown in Table 1 are used. The number of technicians, number of tasks, and the travel and task duration are chosen such that a theoretical mean utilization of $106.25 \%$ is reached (4 tasks per day and 5 journeys), hence, not all tasks can be fulfilled within one day, leading to the prioritization of tasks. Even though this theoretical utilization is higher than reported in literature (e.g. [41]), it represents our findings from practice very well, as maintenance providers always have a backlog of tasks that need to be served. Travel costs are based on billing data of a German medium-sized machine manufacturer offering maintenance services to their customers.

\subsection{Results and Discussion}

The results of the simulation experiment are shown in Table 1. As mentioned, each case was simulated $k=250$ times and the average improvement through the shift from provider- to system-oriented service delivery is given in Table 2 for each factor value combination. Prior to discussing the results, we want to point out that the findings of this work need to-as all results generated by simulation-be handled with care, as simulation studies only test many, yet not all possible instances of a stochastic variable. Hence, only sample of the stochastic possible populations are analyzed.

Table 2. Simulation experiment results: improvement (in \%).

\begin{tabular}{c||c|c|c|c}
\hline Distribution / Variance & 40000 & 90000 & 160000 & 250000 \\
\hline $\mathrm{N}$ & 3.23 & 4.76 & 6.32 & 7.29 \\
$\mathrm{U}$ & 1.28 & 1.34 & 1.68 & 1.52 \\
M2 & 3.09 & 4.97 & 6.50 & 8.52 \\
M5 & 2.99 & 4.78 & 6.57 & 8.61 \\
M10 & 2.85 & 4.68 & 6.60 & 8.64 \\
\hline mean & 2.69 & 4.11 & 5.53 & 6.92 \\
\hline
\end{tabular}

First, the results show that-on average-the added value was greater than 0 , hence, these results align with previous work by Wolff et al. [3]. Of the total of 5000 simulation cases, 203 (4\% of simulation runs) indicate a negative improvement, i.e. a degradation, through the shift from provider- to system-oriented service delivery. In those 203 cases, the average degradation of total delivery-dependent system costs is $0.95 \%$ and can be linked to heuristic solving. Due to heuristic solving, it is possible that non-optimal technician schedules are created, and, thus, result in higher system costs in system-oriented service delivery.

Second-only looking at the mean improvement regardless of the customer base's slope distribution-our results suggest that the added value created through system-oriented service delivery increases with increasing variance of the customer base cost slopes. The mean improvement over all distributions with a variance of 40000, 90000, 160000, and 250000 is $2.69 \%, 4.11 \%, 5.53 \%$, and $6.92 \%$, 
respectively. This supports our initial example that the potential of system-oriented service delivery with two highly different customers is high. However, a more sophisticated analysis is required here.

Third-as displayed in Figure 3-our results indicate that the added value through system-oriented service delivery is-regardless of cost slope variation-the lowest in the case of a customer base with uniformly distributed cost slopes. Furthermore, the results of the simulation experiment suggest that markets with different segments-modeled by the mixture distributions - have the highest gain through system-oriented service delivery. Surprisingly, the degree of segment separability (factor $X$ ) has very little impact on the magnitude of added value created. In our study, the added value for all $X$ was within $0.3 \%$.

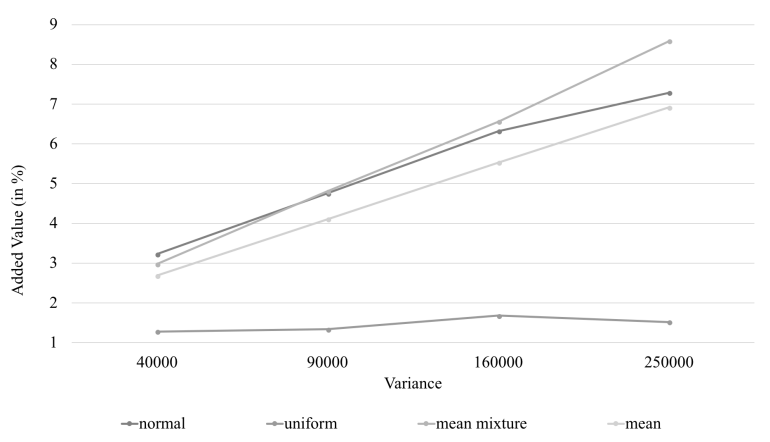

Figure 3. Experiment Results.

Furthermore, we want to point out that the utilization of technicians does not change significantly between the provider- and system-oriented service delivery. This is an important benchmark and indicator that the simulation model itself is valid, as utilization cannot change drastically between the two solutions as the ratio between demand (tasks) and delivery resources (technicians) has not changed.

The low added value in a customer base with uniformly distributed cost slopes can be explained as follows: The uniform distribution results-compared to the other distributions - in a far smaller range of drawn values given the fixed provided overall variance. Consequently, the potential of reducing total costs is low, as customer's cost slopes are fairly close one another.

Our results indicate that the added value-computed based on the comparison against a base case-is the highest in two distinguishable segment markets. However, in practice, the segment with cost slopes may have agreed on certain service levels, and, consequently, requires a different base case. Indeed, it is a very interesting question to see how system-oriented service delivery compares to provider-oriented service delivery if certain service level agreements (e.g. in the form of response time guarantees) and varying penalties are in place. Whilst added value will still created, we believe it to be smaller compared to the findings of this work.

Given those results, we conclude the following with regard to the objectives of this work: First, our findings indicate total system costs savings through system-oriented service delivery. Assuming appropriate compensation methods in place, a shift from provider-oriented to system-oriented service delivery will result in a Pareto improvement. Given this illustrative scenario, cost savings of nearly 9\% are possible, indicating the high potential of system-oriented service delivery. Second, the overall variance of the customers' cost slopes seems to be positively correlated with the added value, hence, supporting previous thoughts on the range of cost slopes influencing the added value. However, more sophisticated analysis is required to further support this indication. Third, regardless of the overall cost slope variance, the distribution of the slope also impacts the added value (ordering: uniform $\leq$ normal $\leq$ mixture).

\section{Conclusion}

In this section, we summarize this work and highlight its contribution. Furthermore, we point out limitations and give directions for further research.

\subsection{Summary and Contribution}

In this work, we present a simulation study to elaborate on the magnitude of added value created through system-oriented service delivery, a novel approach in service delivery aiming at minimizing the delivery-dependent costs over all system participants. The idea is rooted in the argument that all participant have individual and varying costs associated to service delivery. In industrial maintenance, for example, those costs reflect costs of downtime due to unavailable production equipment. Using the simulation study, we elaborate on the impact of different customer bases with regards to their cost slopes (i.e. response time sensitiveness of their costs of downtime). In other words, we use different compositions of customers with regards to their cost increase over response time and measure the impact on the added value created through system-oriented service delivery.

The findings of this work suggest that the distribution of the customers' cost slopes impacts the magnitude of added value through system-oriented service delivery significantly. Uniformly distributed cost slopes (resulting in a small, yet evenly distributed cost slopes) result in much smaller gains through 
system-oriented service delivery compared to normally distributed cost slopes among customers. In a customer base consisting of two distinguishable segments with regard to their cost slopes (modeled by mixture distributions), the added value was the greatest, and, hence, system-oriented service delivery has the highest impact. Furthermore, regardless of cost slope distribution, slope variance seems to be positively correlated with the added value.

By elaborating on the added value created through system-oriented service delivery, this work contributes to the new field of system-oriented service delivery introduced by Wolff et al. [3]. In a broader sense, this work also contributes to the field of service science, service systems, and sharing economy as it demonstrates and try to measure the benefit gained from multiple participants not only interacting with each other, but also sharing benefits and losses among them.

\subsection{Limitations and Future Work}

Even though this work marks an important step towards the introduction of system-oriented service delivery, it certainly has several limitations.

First, the simulation model is based on an illustrative scenario. Even though insights can be generated using an illustrative scenario [6], it still contains assumptions and simplifications. For example, we neglected technician skills and dispatching was done under certainty. Future work can extend this work by adding further complexity by, for example, including technician skills, introduce uncertainty, add customer-dependent service level agreements, extend the planning horizon, or allow for online scheduling. Furthermore, we relied on normally distributed task duration and travel times. Depending on the actual use case, other distributions may be better suited.

Second, we applied a simple model regarding delivery-dependent costs. In industrial maintenance, for example, costs of downtime may not increase linearly as buffer capacities can compensate a short downtime. Furthermore, costs also depend on the current production schedule and production demand at the time of failure. Consequently, more complex models are required in future work.

Third, one must be careful in interpreting the results of the simulation study. As method, simulation has the limitation of only simulating individual samples of a stochastic process, and, hence, no general statements can be made. Even though researchers try to minimize the risk of faulty results by increasing the number of repetitions, results may still not represent reality well.

Fourth, we call for more sophisticated research elaborating on the influence of the cost slope variance on the added value. A thorough understanding of the relationship between the customers' cost slopes on the added value is crucial for the successful implementation of system-oriented service delivery in practice.

Fifth, as indicated by Wolff et al. [3], the question of the distribution of the added value created through system-oriented service delivery remains unanswered. However, this is an important question that need to be addressed for the successful introduction of system-oriented service delivery in practice. During distribution mechanism design, one should incorporate game theoretical approaches and try to align participant individual interests (profit maximization) with system interests (minimal system costs).

Finally, the added value depends on various factors. In this work, we only elaborated on the customer side, namely, their cost slopes. However, the added value is determined by the interchange of multiple variables, as, for example, also the change of provider costs between different delivery solutions and their ratio compared to the customer costs. Therefore, this work only marks first steps into this new field of research and we call for further research elaborating on the added value introduced through system-oriented service delivery.

\section{References}

[1] A. Ostrom, M. Bitner, S. Brown, K. Burkhard, M. Goul, V. Smith-Daniels, H. Demirkan, and E. Rabinovich, "Moving Forward and Making a Difference: Research Priorities for the Science of Service," Journal of Service Research, vol. 13, no. 1, pp. 4-36, 2010.

[2] A. Ostrom, A. Parasuraman, D. Bowen, L. Patrício, and C. Voss, "Service Research Priorities in a Rapidly Changing Context," Journal of Service Research, vol. 18, no. 2, pp. 127-159, 2015.

[3] C. Wolff, N. Kühl, and G. Satzger, "System-Oriented Service Delivery: The Application of Service System Engineering to Service Delivery," in Proceedings of the 26th European Conference on Information Systems, pp.,- 2018.

[4] J. Spohrer and P. Maglio, "Toward a Science of Service Systems," pp. 157-194, Springer US, 2010.

[5] T. Böhmann, J. M. Leimeister, and K. Möslein, "Service Systems Engineering," Business \& Information Systems Engineering, vol. 6, no. 2, pp. 73-79, 2014.

[6] K. Peffers, M. Rothenberger, T. Tuunanen, and R. Vaezi, "Design Science Research Evaluation," in Proceedings of the 7th International Conference on Design Science Research in Information Systems, pp. 398-410, 2012.

[7] R. Gitzel, B. Schmitz, H. Fromm, A. Isaksson, and T. Setzer, "Industrial Services as a Research Discipline," Enterprise Modelling and Information Systems Architectures, vol. 11, no. 1, pp. 1-22, 2016.

[8] W. Geraerds, "The cost of downtime for maintenance : preliminary considerations," EUT - BDK report. Dept. of Industrial Engineering and Management Science, vol. 11, 1984. 
[9] F. Chan, H. Lau, R. Ip, H. Chan, and S. Kong, "Implementation of total productive maintenance: A case study," International Journal of Production Economics, vol. 95, no. 1, pp. 71-94, 2005.

[10] L. Swanson, "Linking maintenance strategies to performance," International Journal of Production Economics, vol. 70, no. 3, pp. 237-244, 2001.

[11] C. Wolff, M. Vössing, B. Schmitz, and H. Fromm, "Towards a Technician Marketplace using Capacity-Based Pricing," in Proceedings of the 51th Hawaii International Conference on System Sciences, pp. 1553-1562, 2018.

[12] S. Huber and S. Spinler, "Pricing of full-service repair contracts," European Journal of Operational Research, vol. 222, no. 1, pp. 113-121, 2012.

[13] B. Schmitz, F. Duffort, and G. Satzger, "Managing Uncertainty in Industrial Full Service Contracts: Digital Support for Design and Delivery," pp. 123-132, 2016.

[14] P. Datta and R. Roy, "Cost modelling techniques for availability type service support contracts: A literature review and empirical study," CIRP Journal of Manufacturing Science and Technology, vol. 3, no. 2, pp. 142-157, 2010.

[15] P. Hypko, M. Tilebein, and R. Gleich, "Benefits and uncertainties of performance-based contracting in manufacturing industries," Journal of Service Management, vol. 21, no. 4, pp. 460-489, 2010.

[16] R. Klimberg and F. Van Bennekom, "Aggregate planning models for field service delivery," Location Science, vol. 5, no. 3, pp. 181-195, 1997.

[17] G. Dantzig and J. Ramser, "The Truck Dispatching Problem," Management Science, vol. 6, no. 1, pp. 80-91, 1959.

[18] J.-F. Cordeau, G. Laporte, F. Pasin, and S. Ropke, "Scheduling technicians and tasks in a telecommunications company," Journal of Scheduling, vol. 13, no. 4, pp. 393-409, 2010.

[19] I. Petrakis, C. Hass, and M. Bichler, "On the impact of real-time information on field service scheduling," Decision Support Systems, vol. 53, no. 2, pp. 282-293, 2012.

[20] A. Kovacs, S. Parragh, K. Doerner, and R. Hartl, "Adaptive large neighborhood search for service technician routing and scheduling problems," Journal of Scheduling, vol. 15, no. 5, pp. 579-600, 2012.

[21] M. Drexl, "Rich vehicle routing in theory and practice," Logistics Research, vol. 5, no. 1, pp. 47-63, 2012.

[22] R. Lahyani, M. Khemakhem, and F. Semet, "Rich vehicle routing problems: From a taxonomy to a definition," European Journal of Operational Research, vol. 241, no. 1, pp. 1-14, 2015.

[23] J. Xu and S. Chiu, "Effective Heuristic Procedures for a Field Technician Scheduling Problem," Journal of Heuristics, vol. 7, no. 5, pp. 495-509, 2001.

[24] S. Ropke and D. Pisinger, "An Adaptive Large Neighborhood Search Heuristic for the Pickup and Delivery Problem with Time Windows," Transportation Science, vol. 40, no. 4, pp. 455-472, 2006.

[25] M. M. Solomon, "Algorithms for the Vehicle Routing and Scheduling Problems with Time Window Constraints," Operations Research, vol. 35, no. 2, pp. 254-265, 1987.
[26] K. Lacommare and J. Eto, "Cost of power interruptions to electricity consumers in the United States (US)," Energy, vol. 31, no. 12, pp. 1845-1855, 2006.

[27] A. Kieninger, F. Berghoff, H. Fromm, and G. Satzger, "Simulation-Based Quantification of Business Impacts Caused by Service Incidents," in Proceedings of the 4th International Conference on Exploring Service Science (IESS), pp. 170-185, 2013.

[28] D. Crumrine and D. Post, "When true cost of downtime is unknown, bad decisions ensue," InTech, vol. 53, no. 1, pp. 55-60, 2006.

[29] A. Salonen and M. Tabikh, "Downtime Costing-Attitudes in Swedish Manufacturing Industry," in Proceedings of the 10th World Congress on Engineering Asset Management (WCEAM 2015), pp. 539-544, 2016.

[30] J. Fox, J. Brammall, and P. Yarlagadda, "Determination of the financial impact of machine downtime on the Australia Post large letters sorting process," in 9th Global Congress on Manufacturing and Management, pp. 732-738, 2008.

[31] D. Edwards, G. Holt, and F. Harris, "Predicting downtime costs of tracked hydraulic excavators operating in the UK opencast mining industry," Construction Management and Economics, vol. 20, no. 7, pp. 581-591, 2002.

[32] S. Vegunta and J. Milanovic, "Estimation of Cost of Downtime of Industrial Process Due to Voltage Sags," IEEE Transactions on Power Delivery, vol. 26, no. 2, pp. 576-587, 2011.

[33] C. Wolff and B. Schmitz, "Determining Cost-Optimal Availability for Production Equipment using Service Level Engineering," in 2017 IEEE 19th Conference on Business Informatics (CBI), pp. 176-185, 2017.

[34] P. Maglio and J. Spohrer, "Fundamentals of service science," Journal of the Academy of Marketing Science, vol. 36, no. 1, pp. 18-20, 2008.

[35] J. Spohrer and P. Maglio, "The Emergence of Service Science: Toward Systematic Service Innovations to Accelerate Co-Creation of Value," Production and Operations Management, vol. 17, no. 3, pp. 238-246, 2008.

[36] S. Vargo, P. Maglio, and M. Akaka, "On value and value co-creation: A service systems and service logic perspective," European Management Journal, vol. 26, no. 3, pp. 145-152, 2008.

[37] P. Maglio and J. Sporen, "A service science perspective on business model innovation," Industrial Marketing Management, vol. 42, no. 5, pp. 665-670, 2013.

[38] A. Law, Simulation modeling and analysis. New York, NY: McGraw-Hill Education, 5. ed., in ed., 2015.

[39] K. Sörensen, M. Sevaux, and P. Schittekat, "Multiple Neighbourhood" Search in Commercial VRP Packages: Evolving Towards Self-Adaptive Methods, pp. 239-253. Berlin, Heidelberg: Springer Berlin Heidelberg, 2008.

[40] R. Sargent, "Verification and validation of simulation models," in Proceedings of the 2009 Winter Simulation Conference (WSC), pp. 162-176, 2009.

[41] E. Watson, P. Chawda, B. McCarthy, M. Drevna, and R. Sadowski, "A Simulation Metamodel for Response-Time Planning," Decision Sciences, vol. 29, no. 1, pp. 217-241, 1998. 